\title{
The Difficult Diagnosis of Temporomandibular Joint Arthritis
}

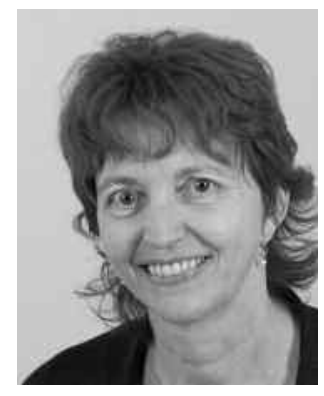

Arthritis of the temporomandibular joint (TMJ) is a long-recognized feature of juvenile idiopathic arthritis (JIA). The consequences of longstanding TMJ inflammation - severe micrognathia and retrognathism - were mentioned in the original description of Sir Frederic Still ${ }^{1}$. Growth problems are common in JIA, but the extent of growth failure seen in the mandible of children with TMJ arthritis by far exceeds arthritis-related growth complications of other locations. They are caused by unique properties of the mandible: in contrast to what one would assume, the mandible does not grow at the chin. The teeth-bearing body of the mandible undergoes only minimal growth during childhood, while the majority of new bone formation takes place on the mandibular ramus and condyle ${ }^{2}$. In fact, the majority of mandibular growth originates from chondrocytes immediately adjacent to the TMJ joint space. Because of this close proximity, the inflammatory cytokines in the synovial fluid may have a direct effect on condylar growth very early on in the event of arthritis $^{3}$. Further, the total growth of the mandible during childhood is considerable, and thus also the potential effect size of disturbed growth of this bone.

Another unique factor of TMJ arthritis in JIA is the absence of clinical signs and symptoms in most patients. In the study of Twilt, et $a l^{4}$, only $12 \%$ of patients had TMJ pain; Billiau, et al found $22 \%$ with muscle or joint tenderness ${ }^{5}$, and Argyropoulou, et al found 5\%-8\% with pain or chewing discomfort ${ }^{6}$. Also, clinical examination is not able to reveal early signs of active TMJ arthritis, even when performed by skilled examiners; whether they are pediatric rheumatologists or orthodontists, they will reliably find only the signs of structural damage (growth delay, asymmetric movement on mouth opening), while early inflammation is missed $^{7}$. Unfortunately, the same is also true for ultrasound examination of the $\mathrm{TMJ}^{7,8}$. Thus, magnetic resonance imaging (MRI) remains the method of choice for making or ruling out the diagnosis of TMJ arthritis in children with JIA. Having to rely on MRI for the diagnosis and treatment con- trol of a condition causes a lot of problems: apart from being expensive and of restricted availability in many places, the necessity of lying motionless during the lengthy examination requires sedation in young children. Especially on the background of a disease with few or no subjective symptoms, the decision to have their child undergo an examination under general sedation is understandably difficult for parents. In older children, the necessity of an intravenous access for application of contrast medium may cause an additional barrier to willingness of patients to undergo this procedure frequently.

The arrival of new treatment options for JIA during the past 2 to 3 decades has led to new treatment strategies, with disease remission now being the goal ${ }^{9}$. As a consequence, the severe sequelae of missed or undertreated TMJ arthritis are no longer considered acceptable and a new awareness of the issue of TMJ involvement in JIA has arisen. Finding factors reliably associated with an increased frequency of TMJ involvement in children with JIA would be most helpful for the development of screening recommendations similar to what we know from ophthalmologic screening examinations.

In this issue of The Journal, Stoll, et al describe their findings in a large cohort of JIA patients with TMJ MRI ${ }^{10}$, the largest cohort reported so far. The study further supports important previous findings: TMJ arthritis in patients with JIA is frequent and is often clinically silent; active TMJ arthritis was found in $43 \%$ of patients examined, and in $37 \%$ of them the clinical examination was completely normal. Other cohorts of unselected patients with JIA showed similar numbers for TMJ involvement ${ }^{4,11}$, whereas in cohorts of patients with newly diagnosed JIA the rate can even exceed $80 \%$ of cases $^{8,12}$. The TMJ can be the only joint where arthritis remains active: $49 \%$ of the patients with active TMJ arthritis had no other joint with active arthritis. Without the MRI their JIA would have been considered inactive or even in remission. This is in keeping

See Risk factors for TMJ arthritis in JIA, page 1880 
with many examples of patients whose TMJ arthritis was missed until severe growth disturbance finally led to the diagnosis. TMJ arthritis may be persistently active despite the use of one or more disease modifying antirheumatic drugs (DMARD): $62 \%$ of the patients with active TMJ arthritis were taking $\geq 1$ DMARD at the time of examination, and 38\% were on a combination therapy of $\geq 2$ DMARD. Although previous studies had demonstrated a beneficial effect of methotrexate for the facial development of patients with JIA ${ }^{13}$, and in the present study the percentage of patients with TMJ arthritis was lower (although not statistically significant), in the cohort with DMARD therapy, the conclusion must be that we cannot call JIA inactive if an MRI scan of the TMJ was not performed.

The most consistent clinical finding in children with TMJ arthritis across all reports is a reduced mouth-opening capacity $4,7,11$. Even patients with early TMJ arthritis without structural damage have significantly reduced mouth-opening compared to healthy controls ${ }^{7}$. It would be intriguing to use mouth-opening capacity to preselect patients with a high probability of TMJ arthritis already before MRI. However, as Stoll, et al demonstrate very nicely, a single mouth-opening measurement is not able to predict or exclude TMJ arthritis because of the large interindividual variability of this measure. Still, one may speculate that maximal mouth-opening capacity may one day be a helpful tool to follow a patient during the JIA disease course, as a decrease from an individual baseline measurement may be a sensitive sign for new onset or a flare of TMJ arthritis. To have age-and sex-related percentiles of mouth-opening capacity - similar to other growth percentiles used in pediatrics would therefore be a most helpful tool.

An important limitation of the current study is the lack of information about the 195 patients who did not have TMJ MRI scans. From the experience of everyday work with JIA patients one would assume that there is almost certainly a bias by indication, but the lack of data about the total cohort makes it impossible to estimate the extent of this bias. However, knowing that the peak age at onset of JIA is around 2 years of age, the relatively old mean age at examination despite a relatively high number of newly diagnosed patients and a short mean disease duration all point towards a bias with fewer MRI scans performed in very young patients who would need sedation.

In contrast to all previous studies, where early disease onset and longer disease duration were associated with TMJ arthritis, Stoll, et al found a higher percentage of TMJ involvement in children with older age at onset and shorter disease duration. These findings must be carefully weighed: most studies rely on conventional radiographs and/or clinical signs for the diagnosis of TMJ involvement. The diagnosis of TMJ involvement with these modalities actually relies on structural damage caused by TMJ arthritis. As accrual of damage increases with longer disease duration, a combination of early diagnosis and long disease duration would be expected to be associated with TMJ damage. In contrast, in the current study the diagnosis was based on MRI findings, a method highly sensitive for inflammatory signs even without structural damage. While nearly every patient with TMJ involvement had signs of active inflammation, only $27 \%$ had chronic changes. Also, the disease duration was shorter, with a mean of only 2.5 years, compared to 6.9 years in the study of Cannizzaro, et $a l^{11}$ and 5 years in the study of Twilt, et $a l^{4}$. In addition, there was a high percentage of patients with $<3$ months' disease duration, who inevitably have a higher chance of active disease because they may not yet have started treatment, or their systemic medication may not have had time to develop its full effect. In summary, the seeming contradictions with results of previous studies may be related to the characteristics of the cohort (possible bias towards inclusion of older children not needing sedation; short disease duration) and the diagnostic method used (MRI).

The study of Stoll, et al very precisely shows why a single measurement of mouth-opening will not help to distinguish between patients with and those without TMJ involvement. It confirms some previous findings while questioning others, and leaves us with the insight that TMJ arthritis remains a problem in children with JIA, and we are only just discovering how much we don't know about it.

ROTRAUD SAURENMANN, MD, Head, Pediatric Rheumatology, University Children's Hospital, Steinwiesstrasse 75, CH-8032 Zurich, Switzerland

Address correspondence to Dr. Saurenmann;

E-mail: traudel.saurenmann@kispi.uzh.ch

\section{REFERENCES}

1. Still GF. On a form of chronic joint disease in children. Medico.Chirurgical Transactions 1896;80:47-59.

2. Bjork A, Skieller V. Normal and abnormal growth of the mandible. A synthesis of longitudinal cephalometric implant studies over a period of 25 years. Eur J Orthod 1983;5:1-46.

3. Pirttiniemi P, Peltomaki T, Muller L, Luder HU. Abnormal mandibular growth and the condylar cartilage. Eur J Orthod 2009;31:1-11.

4. Twilt M, Mobers SM, Arends LR, ten Cate R, van Suijlekom-Smit L. Temporomandibular involvement in juvenile idiopathic arthritis. J Rheumatol 2004;31:1418-22.

5. Billiau AD, Hu Y, Verdonck A, Carels C, Wouters C. Temporomandibular joint arthritis in juvenile idiopathic arthritis: Prevalence, clinical and radiological signs, and relation to dentofacial morphology. J Rheumatol 2007;34:1925-33.

6. Argyropoulou MI, Margariti PN, Karali A, Astrakas L, Alfandaki S, Kosta P, et al. Temporomandibular joint involvement in juvenile idiopathic arthritis: Clinical predictors of magnetic resonance imaging signs. Eur Radiol 2009;19:693-700.

7. Muller L, Kellenberger CJ, Cannizzaro E, Ettlin D, Schraner T, Bolt IB, et al. Early diagnosis of temporomandibular joint involvement in juvenile idiopathic arthritis: A pilot study comparing clinical 
examination and ultrasound to magnetic resonance imaging. Rheumatology 2009;48:680-5.

8. Weiss PF, Arabshahi B, Johnson A, Bilaniuk LT, Zarnow D, Cahill AM, et al. High prevalence of temporomandibular joint arthritis at disease onset in children with juvenile idiopathic arthritis, as detected by magnetic resonance imaging but not by ultrasound. Arthritis Rheum 2008;58:1189-96.

9. Beukelman T, Patkar NM, Saag KG, Tolleson-Rinehart S, Cron RQ, DeWitt EM, et al. 2011 American College of Rheumatology recommendations for the treatment of juvenile idiopathic arthritis: Initiation and safety monitoring of therapeutic agents for the treatment of arthritis and systemic features. Arthritis Care Res 2011;63:465-82.

10. Stoll ML, Sharpe T, Beukelman T, Good J, Young D, Cron RQ Risk factors for temporomandibular joint arthritis in children with juvenile idiopathic arthritis. J Rheumatol 2012;39:1880-7.
11. Cannizzaro E, Schroeder S, Muller LM, Kellenberger CJ, Saurenmann RK. Temporomandibular joint involvement in children with juvenile idiopathic arthritis. J Rheumatol 2011;38:510-5.

12. Kuseler A, Pedersen TK, Herlin T, Gelineck J. Contrast enhanced magnetic resonance imaging as a method to diagnose early inflammatory changes in the temporomandibular joint in children with juvenile chronic arthritis. J Rheumatol 1998;25:1406-12.

13. Ince DO, Ince A, Moore TL. Effect of methotrexate on the temporomandibular joint and facial morphology in juvenile rheumatoid arthritis patients. Am J Orthod Dentofacial Orthop 2000;118:75-83.

J Rheumatol 2012;39:1778-80; doi:10.3899/jrheum.120635 